\title{
Acetaldehyde adducts in alcoholic liver disease
}

\author{
Mashiko Setshedi, ${ }^{1,3}$ Jack R. Wands' and Suzanne M. de la Monte ${ }^{1,2, *}$ \\ 'Department of Medicine; 'Department of Pathology; Rhode Island Hospital and the Alpert Medical School of Brown University; Providence, Rl; \\ ${ }^{3}$ Department of Internal Medicine; University of Cape Town; Cape Town, South Africa
}

Key words: alcohol, cirrhosis, hepatocellular carcinoma, cancer, alcohol metabolism, acetaldehyde, adducts, liver disease, cardiovascular disease, atherosclerosis

Chronic alcohol abuse causes liver disease that progresses from simple steatosis through stages of steatohepatitis, fibrosis, cirrhosis, and eventually hepatic failure. In addition, chronic alcoholic liver disease (ALD), with or without cirrhosis, increases risk for hepatocellular carcinoma (HCC). Acetaldehyde, a major toxic metabolite, is one of the principal culprits mediating fibrogenic and mutagenic effects of alcohol in the liver. Mechanistically, acetaldehyde promotes adduct formation, leading to functional impairments of key proteins, including enzymes, as well as DNA damage, which promotes mutagenesis. Why certain individuals who heavily abuse alcohol, develop HCC (7.2-15\%) versus cirrhosis (15-20\%) is not known, but genetics and co-existing viral infection are considered pathogenic factors. Moreover, adverse effects of acetaldehyde on the cardiovascular and hematologic systems leading to ischemia, heart failure, and coagulation disorders, can exacerbate hepatic injury and increase risk for liver failure. Herein, we review the role of acetaldehyde adducts in the pathogenesis of chronic ALD and HCC.

\section{Introduction}

Chronic alcohol abuse causes liver pathology that progresses from simple steatosis through stages of steatohepatitis, followed by fibrosis, then cirrhosis, and finally end-stage liver disease. In addition to liver degeneration, chronic alcohol abuse serves as a potent co-factor in the pathogenesis of hepatocellular carcinoma (HCC). Besides liver, chronic alcohol abuse causes permanent injury to the brain, leading to cognitive-motor impairments and neurodegeneration, the cardiovascular system, resulting in heart failure and enhanced atherosclerosis, and various other organs in which it serves as a co-factor in malignant transformation. However, the toll of chronic alcohol abuse is heaviest in liver and brain.

Alcoholic liver disease (ALD) accounts for $40 \%$ of deaths due to cirrhosis, and $28 \%$ of all liver disease related deaths in the US. ${ }^{1}$ These figures correspond to $3.2-3.5 \%$ of all deaths, and $3.6 \%$ of all cancer deaths globally. ${ }^{2,3}$ The risk of developing hepatocellular

*Correspondence to: Suzanne M. de la Monte;

Email:Suzanne_DeLaMonte_MD@Brown.edu

Submitted: 04/12/10; Revised: 05/07/10; Accepted: 05/10/10

Previously published online:

www.landesbioscience.com/journals/oximed/article/12288 carcinoma (HCC) increases with dose of chronically consumed ethanol ${ }^{1,4}$ and alcohol consumption in excess of $80 \mathrm{~g} /$ day is correlated with high rates $(7.2-15 \%)$ of HCC, especially in the setting of cirrhosis. ${ }^{1,3,5-7} \mathrm{HCC}$ is the fifth most common malignancy, with an estimated half million new cases diagnosed worldwide each year. Prognosis is generally poor (median survival $1-2$ months) ${ }^{8}$ due to late discovery and limited effective therapeutic options. Only one-third of patients with HCC are deemed suitable for curative procedures, many of which have not yet been fully validated. ' Given the role of alcohol abuse in the pathogenesis of both liver degeneration and HCC, along other major untreatable and irreversible diseases, including those that afflict the brain and cardiovascular system, it is imperative that we improve our understanding of how alcohol mediates its adverse effects.

\section{Alcohol Metabolism}

Alcohol is detoxified and eliminated primarily in the liver via a series of oxidative metabolic reactions. ${ }^{10,11}$ The three major steps are: (1) reversible oxidation of ethanol to acetaldehyde, which is toxic; (2) non-reversible metabolism of the toxic acetaldehyde to acetate; and (3) breakdown of acetate to water and carbon dioxide (Fig. 1). The first step in alcohol oxidative metabolism is effectuated by key enzymes, including alcohol dehydrogenase (ALD), cytochrome P450 2E1 (CYP2E1), and catalase. ADH is the main oxidizing enzyme; it has a high affinity for alcohol ${ }^{12}$ and breaks down ethanol in the cytoplasm. CYP2E1 is utilized by a distinct pathway that is induced by chronic alcohol consumption, and results in acetaldehyde formation in peroxisomes. A third path of first-step ethanol metabolism is mediated by catalase oxidation of ethanol in microsomes. ${ }^{13,14}$

The second step, which is mainly carried out by mitochondrial aldehyde dehydrogenase (ALDH), is to metabolize acetaldehyde to acetate. In addition, acetaldehyde can be metabolized by CYP2E1 through an NADPH-dependent pathway (microsomal acetaldehyde-oxidizing system). ${ }^{15}$ The resulting acetate is unstable and spontaneously breaks down to water and $\mathrm{CO}_{2}$. When these oxidative mechanisms become overwhelmed, acetaldehyde accumulates and exerts its toxic effects. The electrophilic nature of acetaldehyde ${ }^{12}$ enables it to bind and form adducts, i.e. covalent chemicaladdition products, with proteins, lipids, andDNA., $311,16-18$ Adducts are broadly pathogenic because they impair functions of proteins and lipids, and promote DNA damage and mutation. ${ }^{11}$ 


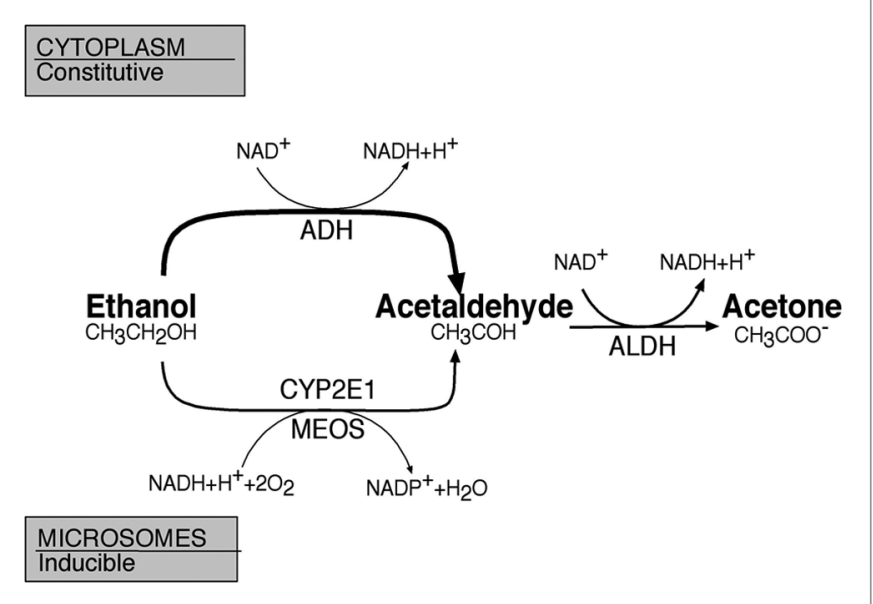

Figure 1. Metabolic pathways of alcohol. The main oxidizing enzyme is cytosolic alcohol dehydrogenase (ADH), which converts ethanol to acetaldehyde. Cytochrome P450 2E1 (CYP2E1), an enzyme of the microsomal ethanol oxidizing system (MEOS) that is inducible with chronic alcohol consumption also metabolizes alcohol. The conversion of acetaldehyde (a toxic metabolite) to acetate is by mitochondrial aldehyde dehydrogenase (ALDH). Both ADH and ALDH have polymorphisms that affect the rate at which acetaldehyde is generated and metabolized.

\section{Alcohol Enzyme Polymorphisms}

Propensity for acetaldehyde accumulation vis-à-vis ethanol consumption is governed in part by genetic factors. Polymorphisms in the ADH and ALDH genes can affect the rates of acetaldehyde generation and metabolism, and therefore govern proneness to acetaldehyde toxicity. In humans, there are at least 8 iso-enzymes of ADH and 4 iso-enzymes of ALDH. The most important $\mathrm{ADH}$ iso-enzymes are those encoded by the ADH1A, $\mathrm{ADH} 1 \mathrm{~B}$ and $\mathrm{ADH} 1 \mathrm{C}$ genes. ${ }^{3,19}$ In addition, various $\mathrm{ADH} 1 \mathrm{~B}$ and ADH1C alleles account for differences in the activity of ADH. For example, the ADH1B*2 allele encodes an enzyme that is 40 times more active than the one encoded by the $\mathrm{ADH} 1 \mathrm{~B}^{*} 1$ allele. Rapid metabolism of alcohol to acetaldehyde, in individuals who express the $\mathrm{ADH}_{1} \mathrm{~B}^{*} 2$ allele, results in its toxic accumulation. The clinical manifestations of this effect include, flushing, sweating, tachycardia, nausea and vomiting after ethanol consumption. This $\mathrm{ADH}_{1} \mathrm{~B}^{*} 2$ allele is predominantly found in Asian populations. Although short-term responses to ethanol cause considerable discomfort, the adverse physiological response could serve as a deterrent for chronic alcohol misuse. The $\mathrm{ADH} 1 \mathrm{C}^{*} 1$ allele is another ADH iso-enzyme with increased (2.5-fold) activity, but the physiological effects associated with alcohol consumption are modest compared with those of ADH1B*2. ${ }^{3}$ Nonetheless, individuals carrying at least one $\mathrm{ADH} 1 \mathrm{C}^{*} 1$ allele are at increased risk (3.6-fold) for developing alcohol-related HCC compared with those who are homozygous for $\mathrm{ADH} 1 \mathrm{C}^{*} 2 .^{20}$ This suggests that genetic polymorphisms in $\mathrm{ADH}$ that result in increased generation of acetaldehyde also enhance ethanol's pro-carcinogenic effects.

ALDH2 is encoded by two main alleles, ALDH2*1 and ALDH2*2. ALDH $2 * 2$ is an inactive enzyme, and when expressed, acetaldehyde accumulation can be 10- or 20-fold higher than when the ALDH $2 * 1$ active enzyme is expressed, rendering alcohol consumption virtually intolerable. ${ }^{21}$ The ALDH2*2 enzyme is not found in Caucasians, but is homozygous in $10 \%$, and heterozygous in $40 \%$ of Asians. ${ }^{3}$ Low ALDH activity increases risk for aero-digestive cancers, ${ }^{22}$ and among Japanese people, the phenotype increases the risk for malignancy in general. ${ }^{23,24}$ Together, the findings indicate that specific genetic polymorphisms in the ADH and ALDH genes have important roles in increasing susceptibility to alcohol-related cancers among heavy drinkers, and that the adverse effects of ethanol are likely mediated through the toxic and mutagenic effects of acetaldehyde.

\section{Roles of Alcohol and Acetaldehyde in Carcinogenesis}

Experimental animal models have provided convincing evidence that alcohol is a mutagen, ${ }^{3}$ and that acetaldehyde should be regarded as a carcinogen (Table 1). ${ }^{10,11,25,26}$ The role of alcohol/ acetaldehyde as a carcinogen is particularly relevant to the pathogenesis of upper aero-digestive tract cancers. ${ }^{3,27}$ Mechanistically, alcohol mediates its mutagenic effects through: (1) formation of acetaldehyde adducts; ;,28 (2) increased oxidative stress; ;9,30 (3) induction of Kupffer cells by gut-derived endotoxins and release of TNF- $\alpha ;^{31-33}$ (4) inhibition of DNA methylation; and (5) impairing retinoid metabolism, which is important in cell differentiation. ${ }^{1}$ Iron acts either independently ${ }^{34,35}$ or synergistically ${ }^{21,34,36}$ to promote the toxic and mutagenic effects of acetaldehyde. It is noteworthy that similar mechanisms contribute to the pathogenesis of chronic alcohol-induced liver injury leading to fibrosis or cirrhosis.

Acetaldehyde exerts its mutagenic effects by interacting directly with DNA, ${ }^{37}$ and causing lesions ranging from point mutations to more extensive chromosomal damage. ${ }^{22}$ For example, acetaldehyde-induced point mutations in the hypoxanthine phosphoribosyltransferase gene (HPRT1) impair DNA synthesis, DNA repair mechanisms, particularly nucleotide excision and excision- repair processes that maintain stability and integrity of genomic DNA. ${ }^{37}$ In addition, acetaldehyde causes DNA damage by inducing sister chromatid exchanges (SCE's). ${ }^{16,22}$ Any one of these mutagenic effects can activate cancer-generating pathways, or contribute to liver injury leading to cirrhosis.

\section{Acetaldehyde as an Indirect Carcinogen}

(1) Acetaldehyde protein adducts. Acetaldehyde impairs cellular functions and gene expression by forming adducts with proteins and DNA.11,16,38,39 Acetaldehyde produces protein adducts by interacting with the epsilon amino group of lysine, or the $\alpha$ amino group of $\mathrm{N}$-terminal amino acids. ${ }^{12}$ Stable acetaldehyde adducts alter the structure and function of proteins, including enzymes. For example, acetaldehyde adducts formed with O6 methylguanine methyltransferase, ${ }^{22}$ impair DNA repair mechanisms, which could mediate carcinogenesis. ${ }^{40}$ Other major proteins targeted for acetaldehyde adduct formation include, tubulin, ${ }^{41}$ collagen, ${ }^{42}$ ketosteroid reductase 
Table 1. Mechanisms of tissue injury: Ethanol, acetaldehyde and lipid peroxidation adducts

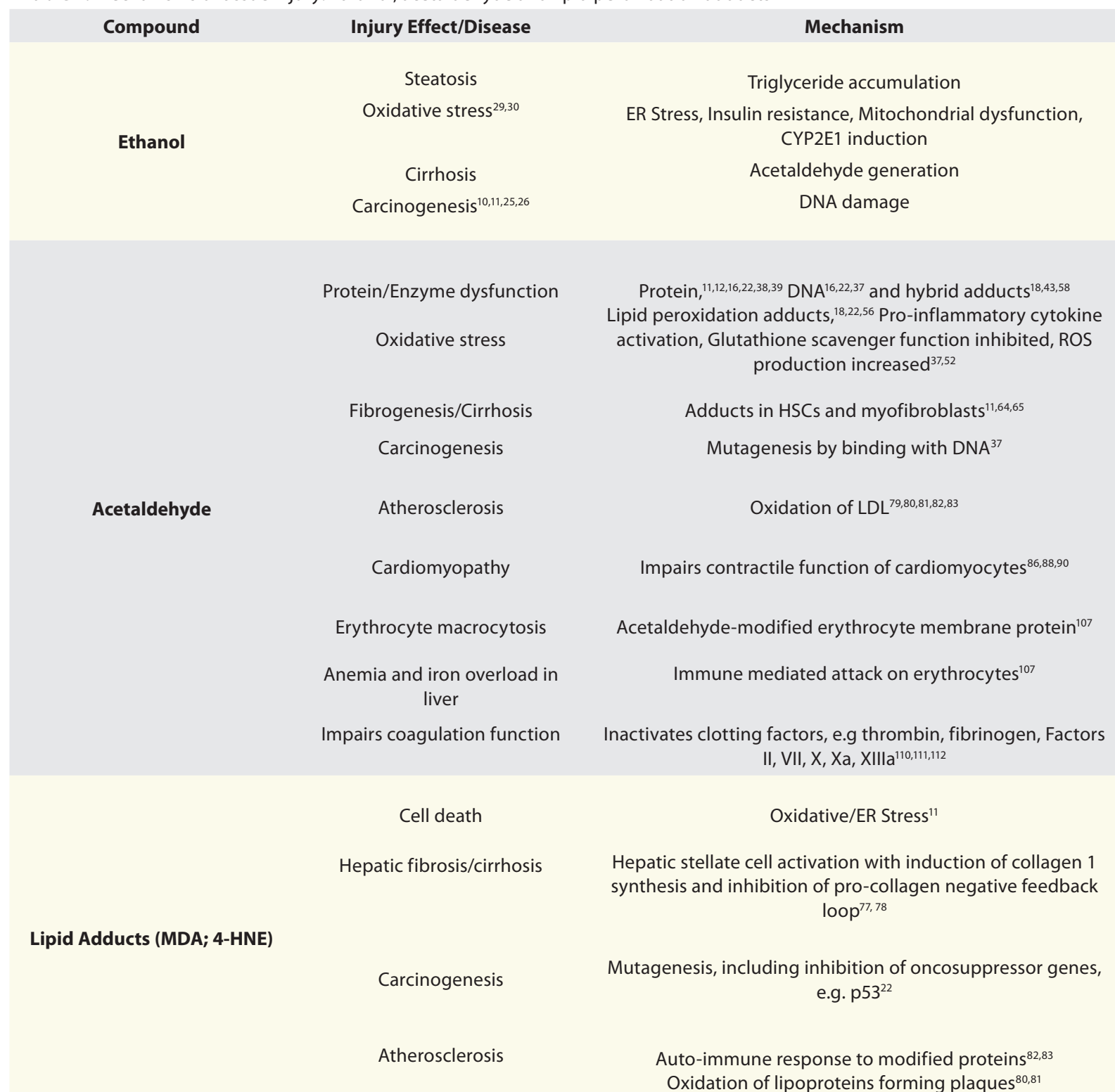

\begin{abstract}
Abbreviations: ER stress, endoplasmic reticulum stress; CYP2E1, cytochrome P450 2E1; ROS, reactive oxygen species; HSCs, hepatic stellate cells; LDL, low-density lipoproteins; MDA, malondialdehyde; 4-HNE, 4-hydroxynonenal.
\end{abstract}

(catalyzes reduction of key intermediates in bile acid biosynthesis), CYP2E $1,{ }^{43}$ and coagulation factors 7 and $9 .{ }^{44}$ Protein adducts impair catalytic activity, ${ }^{45}$ and consequently, functional impairment of CYP2E1 (NADPH-dependent) could lead to further acetaldehyde accumulation. Acetaldehyde binding to glutathione inhibits hydrogen peroxide scavenger function, thereby aggravating oxidative stress and lipid peroxidation. ${ }^{46}$ Lipid peroxidation is probably the most important mediator of alcohol-induced cirrhosis and carcinogenesis. ${ }^{31,47}$

(2) Acetaldehyde DNA adducts. Acetaldehyde generates DNA adducts, the most prevalent of which is N2-ethyldeoxyguanosine $\left(\mathrm{N}^{2}-\mathrm{Et}-\mathrm{dG}\right){ }^{22,37} \mathrm{~N}^{2}-\mathrm{Et}-\mathrm{dG}$ is detectable in livers of alcohol-exposed mice, leukocytes of human alcohol abusers, ${ }^{16,22,48}$ and in humans with an ALDH2 genotype. ${ }^{49,50}$
Due to its stability, $\mathrm{N}^{2}$-Et-dG is detectable in alcohol-associated head and neck cancers,$^{51}$ and therefore could potentially serve as a marker of alcohol mis-use. 1,N(2)-propano-2'-deoxyguanosine (PdG), another acetaldehyde-DNA adduct, is distinguished by its genotoxic and mutagenic effects, and capacity to generate secondary lesions such as DNA-protein and DNA inter-strand cross-links ${ }^{16}$ which impair DNA replication, thereby promoting cell death. Acetaldehyde-DNA adducts also promote carcinogenesis by triggering replication errors and mutations in oncogenes or onco-suppressor genes. ${ }^{22}$

(3) Lipid peroxidation adducts. Alcohol's mutagenic effects can be mediated by induction of CYP2E1, ${ }^{22,52}$ which results in increased generation of reactive oxygen species (ROS) ${ }^{37,52}$ leading to oxidative stress and cell death. ${ }^{11}$ ROS-generated radicals, 
including superoxide anion and hydroxyethyl radical (HER), are highly reactive and form adducts with lipids, proteins and DNA. Hydrogen peroxide, also generated through CYP2E1 enzymatic activity, can react with metal ions such as iron, to produce hydroxyl radicals. ${ }^{37,53}$ Since chronic alcohol abuse causes iron to accumulate in liver, increases in CYP2E1 activity and $\mathrm{H}_{2} \mathrm{O}_{2}$ production can independently or synergistically exacerbate alcohol-induced liver injury and possibly liver cancer via increased hydroxyl radical formation, ${ }^{21,36}$ and attendant DNA strand breakage, as well as a broad range of adverse biological responses. ${ }^{37,54}$

ROS promotes formation of lipid peroxidation products, including malondialdehyde (MDA) and 4-hydroxynonenal (4-HNE), both of which are detectable in association with intense CYP2E1 immunoreactivity in oral squamous cell carcinoma or leukoplakia in alcoholics. ${ }^{55} \mathrm{MDA}$ and 4-HNE can react with DNA bases to form exocyclic DNA adducts. In this regard, MDA reacts with deoxy-guanosine residues, while 4-HNE reacts with deoxyadenosine and deoxycytidine, ${ }^{22,37}$ yielding the adducts, 1,N6-ethenodeoxyadenosine ( $\varepsilon \mathrm{dA})$ and 3,N4-ethenodeoxycytidine $(\varepsilon \mathrm{dC})$, which have important roles in the pathogenesis of chronic alcohol-related liver injury. ${ }^{18,56}$ In addition, both $\varepsilon \mathrm{dA}$ and $\varepsilon \mathrm{dC}$ are highly mutagenic, and can cause mutations in the p53 gene. ${ }^{22}$ Specifically, these adducts induce G:C to T:A transversions on codon 249 , and $30-40 \%$ of HCCs contain a mutation of $\mathrm{p} 53 .{ }^{57}$

(4) Hybrid adducts. Various types of aldehydes generated within cells can cross-react to form hybrid adducts. For example, MAA hybrid adducts are composed of different combinations of MDA-acetaldehyde-protein adducts. The clinical significance of this phenomenon is that hybrid adducts can act synergistically and potentiate carcinogenic potential of individual adducts. ${ }^{18,58}$ In addition, hybrid adducts may mediate stabilization of protein adducts ${ }^{43}$ thereby perpetuating their genotoxic effects.

\section{Adducts Promote Liver Disease}

Adducts accumulate in perivenous regions (Zone 3 ) of rat ${ }^{59,60}$ and human $^{5,61}$ livers, overlapping with the distribution of fatty change (steatosis), i.e. the earliest lesion in alcohol-induced liver injury, ${ }^{62}$ and associated with increased serum aminotransferase levels. ${ }^{63}$ Acetaldehyde protein adducts are detectable in alcohol-related disease-associated inflammation and fibrosis. ${ }^{11,64}$ Ultrastructural and immunohistochemical staining methods revealed that acetaldehyde adducts are detectable in hepatic stellate cells (HSC) in the context of steatofibrosis or cirrhosis, and in myofibroblasts in zones with bridging fibrosis. ${ }^{65}$ Liver injury is likely mediated by binding of acetaldehyde to lysine residues and secondary interference with lysine-dependent enzymes such as calmodulin, and also tubulin. ${ }^{11}$ Acetaldehyde adduct formation with $5 \%$ or less of the available $\alpha$-tubulin pools impairs microtubular function and causes derangement of the hepatocyte cytoarchitecture, as has already been described in ALD. ${ }^{11}$ Similarly, accumulation of acetaldehyde and MDA adducts on areas of collagen deposits contributes to the formation of scar tissue, and subsequent hepatic fibrosis or cirrhosis. ${ }^{11}$
Aldehyde-protein adducts and hydroxyl radicals can cause liver injury by stimulating intense immune responses directed against the modified proteins, as demonstrated by antibodies detected in sera of chronic alcohol-exposed experimental animals $^{66}$ and humans. ${ }^{11}$ Sera of heavy drinkers may contain high titers of $\operatorname{IgM}, \operatorname{IgG}$ and $\operatorname{Ig} A$ antibodies to acetaldehyde adducts. ${ }^{67-69}$ Associated "auto-immune" attacks on hepatocytes cause necrosis, ${ }^{70}$ and with continued rounds of inflammation, necrosis, oxidative stress, ROS generation, and further adduct formation, fibrosis ensues. ${ }^{71}$ An assay for detecting IgA antibodies to acetaldehyde-protein adducts has been developed and can be used to monitor liver injury associated with moderate to heavy drinking. ${ }^{69,72}$

Chronic alcohol abuse leads to progressive liver disease through stages of simple steatosis, to steatohepatitis, fibrosis, and finally cirrhosis through activation of hepatic stellate cells (HSCs). Stellate cells are activated by oxidative stress and lipid peroxidation. ${ }^{73}$ MAA adduct treatment of cultured hepatic endothelial cells stimulates secretion of cytokines and chemokines, including tumor necrosis factor- $\alpha$ (TNF- $\alpha$ ), monocyte chemoattractant protein-1 (MCP-1) and macrophage inflammatory protein-2 (MIP-2) ${ }^{74}$ In addition, MAA adducts activate HSCs by stimulating secretion of fibronectin, which leads to increased extracellular matrix deposition with attendant fibrosis, and eventually cirrhosis. $^{75}$ Mechanistically, adducts generated by alcohol metabolism and lipid peroxidation increase collagen mRNA and connective tissue protein expression. ${ }^{43,76}$ Specifically, acetaldehyde induces collagen 1 synthesis in HSCs by activating the AP-1 transcription factor. ${ }^{77}$ Protein kinase $\mathrm{C}$ phosphorylates $\mathrm{p} 70$ S6k, which promotes protein synthesis and collagen deposition. Acetaldehyde also inhibits the negative feedback loop of procollagen by binding to the carboxyl-terminal propeptide, ${ }^{78}$ further promoting deposition of fibrous matrix. Conversely, adduct scavengers such as chlormethiazole, abolish acetaldehyde-mediated fibrogenesis. ${ }^{43}$ In essence, acetaldehyde adducts contribute to injury, degeneration, scarring and carcinogenesis, and therefore play key roles in the pathogenesis of various stages of alcohol-related liver disease. ${ }^{3}$

\section{Acetaldehyde Effects on the Cardiovascular System: Contribution to Liver Injury}

While moderate consumption of alcohol has cardio-protective effects, excessive amounts worsen atherosclerosis and increase cardiovascular risk. Atherosclerotic plaques form in response to intimal accumulation of oxidized low-density lipoproteins (LDL) in foam cells, which promote oxidative stress and inflammation. ${ }^{79}$ Since aldehyde adducts can mediate oxidation of lipids, including LDL, they potentially have an important role in the pathogenesis of atherosclerosis. Malondialdehyde (MDA), one of the oxidative products of unsaturated fatty acids and a component of atherosclerotic plaques, can also modify LDL. Moreover, both MDA and acetaldehyde can react with various proteins, including apolipoprotein B-100, a component of oxidized LDL, and generate additional adducts that cause endothelial cells to produce and release of pro-inflammatory cytokines and adhesion 


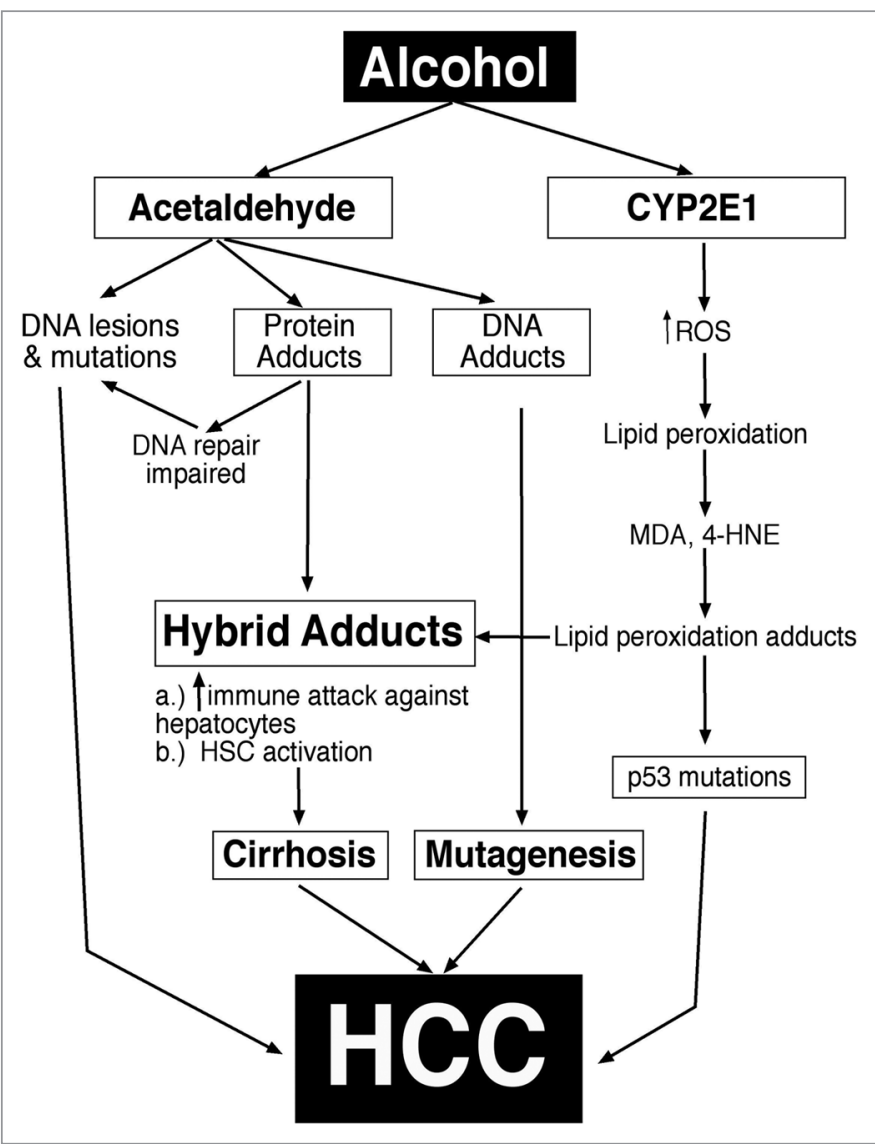

Figure 2. Proposed schematic of alcohol-mediated pathways resulting in hepatocellular carcinoma (HCC). The metabolism of alcohol generates acetaldehyde which promotes carcinogenesis pathways. Acetaldehyde (AA) directly causes DNA lesions, resulting in mutations. Indirectly AA forms covalent bonds with proteins, impairing DNA repair mechanisms, and increasing carcinogenesis potential. AA also binds to DNA, forming DNA adducts (N2-Et-dG and PdG), which are mutagenic. Chronic alcohol consumption results in CYP2E1 induction, generating reactive oxygen species (ROS), thereby increasing lipid peroxidation, which generates MDA and 4-HNE. These lipid peroxidation products cause $\mathrm{p} 53$ mutations and form adducts with DNA. AA-protein adducts combine with DNA adducts to form hybrid adducts, with resultant synergistic activation of auto-immune attacks against hepatocytes, ultimately resulting in cirrhosis, which is strongly associated with HCC development.

molecules that are critical mediators of atherosclerosis. ${ }^{80,81}$ Correspondingly, atherosclerosis can be inhibited by treatment with recombinant antibodies to oxidized $\mathrm{LDL}^{82}$ depleting $\mathrm{B}$ cells that produce autoantibodies to oxidized $\mathrm{LDL}^{83}$ or administration of aldehyde scavengers. ${ }^{84}$ It is noteworthy that MAA adducts are increased in human atherosclerotic vessels ${ }^{80}$ and like MDA, MAA's pro-inflammatory properties can exacerbate atherosclerosis. ${ }^{80}$ Acetaldehyde toxicity and vascular degeneration can contribute to liver disease by causing chronic ischemic injury mediated by atherosclerosis of the aorta and hepatic artery.

Acetaldehyde quite likely has a pathogenic role in alcohol-associated cardiomyopathy. ${ }^{85-89}$ In this regard, it is noteworthy that in experimental animal models, acetaldehyde accumulation in myocardium impairs contractile function, ${ }^{86,88,90}$ disrupts cardiac excitation-contraction coupling $^{87,88,91}$ and promotes oxidative stress ${ }^{85}$ with attendant increased lipid peroxidation. ${ }^{87}$ Acetaldehyde inhibits protein metabolism and induces protein adducts, which together impair the integrity of actin and myosin filaments and depress cardiomyocyte contractile function. In addition, alcohol abuse leads to hypertension, cardiac arrhythmias, and nonischemic cardiomyopathy. ${ }^{92}$ Consequential reductions in cardiac output would lead to liver injury due to chronic passive congestion, and also exacerbate injurious effects of chronic ethanol exposure or acetaldehyde formation. On the other hand, ALDH2 is cardioprotective against ischemic heart disease ${ }^{93}$ and acute cardiac toxicity ${ }^{94}$ because it promotes rapid detoxification of acetaldehyde. Correspondingly, genetic polymorphisms that reduce ALDH2 enzymatic activity are associated with increased rates of diabetes mellitus among Chinese women with coronary artery disease (CAD), independent of alcohol intake, ${ }^{95}$ while over-expression of ALDH2 in transgenic mice reduces alcohol-induced myocardial hypertrophy and impairments in insulin/Akt signaling. ${ }^{9-98}$ Therefore, acetaldehyde can promote cardiovascular disease by several mechanisms including impairment of insulin signaling and cardiomyocyte function, and inducing atherosclerosis, which together could lead to liver injury mediated by ischemia or chronic passive congestion.

The relationship between cardiovascular disease and liver disease is particularly evident in regard to non-alcoholic fatty liver disease (NAFLD). Like alcoholic liver disease, NAFLD progresses through stages of increased inflammation with cell turnover and loss (non-alcoholic steatohepatitis; NASH), fibrosis, cirrhosis, and finally end-stage liver disease. ${ }^{99}$ In addition, progression of NAFLD increases ones risk for developing HCC. ${ }^{100}$ NAFLD is increasingly regarded as the hepatic component driving the increased risk of cardiovascular disease in individuals with diabetes mellitus, hyperlipoproteinaemia, or metabolic syndrome. ${ }^{101-104}$ Moreover, obesity and excess dietary fat intake, which are largely responsible for the epidemic growth in NAFLD, promote systemic oxidative stress and lipid peroxidation, ${ }^{105}$ thereby contributing to the increased risk of cardiovascular disease. Like ALD, progression of NAFLD is associated with increased formation of adducts, including acetaldehyde and MAA, and MAA adduct accumulation correlates with severity of NASH. ${ }^{106}$ Therefore, NAFLD-mediated increases in acetaldehyde and hybrid adducts can contribute to progression of alcoholic liver disease via increased atherosclerosis.

\section{Acetaldehyde Effects on Erythrocytes and Clotting Mechanisms}

Chronic alcohol abuse has significant adverse effects on the hematopoietic system including erythrocyte and coagulation functions. Chronic alcohol abuse promotes red blood cell morphological changes such as erythrocyte macrocytosis. ${ }^{72,107}$ This abnormality has been correlated with the presence of 
autoantibodies to acetaldehyde-modified erythrocyte membrane proteins in peripheral blood ${ }^{107}$ and bone marrow aspirates ${ }^{108}$ Potential consequences of these immune-mediated attacks on erythrocytes are not completely known, but they may increase red blood cell destruction, and thereby promote both anemia and iron accumulation in liver. Excessive iron accumulation is a well-recognized mediator of liver injury and contributes to the pathogenesis of alcoholic liver disease. Moreover, acetaldehydebound to red blood cells can be distributed to various tissues and exert widespread toxic effects. ${ }^{109}$ Finally, a broad range of hematologic disorders can adversely affect liver function. For example, hemolytic anemias and hemaglobinopathies can result in increased iron load in the liver, as well as promote cholelithiasis due to increased formation of bilirubin stones. Alcoholic and other forms of chronic liver disease impair function of coagulation factors, but with regard to alcohol abuse, acetaldehyde mediates these effects by inactivating thrombin, Factor Xa, fibrinogen, II, VII, and X. ${ }^{110-112}$ In addition, acetaldehyde inhibits the transglutaminase activity of factor XIIIa, ${ }^{112}$ and forms complexes with glycosaminoglycans to synergistically inhibit factors IX, IXa, ${ }^{111} \mathrm{X}$, and $\mathrm{Xa},{ }^{110}$ with consequential prolongation of clotting times.

\section{Conclusions}

The data strongly support the concept that chronic and excessive alcohol consumption contributes to and probably promotes progressive liver disease and HCC. These effects of alcohol are mainly mediated by acetaldehyde, which is generated during metabolism of alcohol, and accumulates consequential to genetic polymorphisms in alcohol metabolizing enzymes, increased oxidative stress, iron deposition in liver, and immune-mediated attacks on adducted proteins. Acetaldehyde-protein and DNA adducts promote oxidative stress, formation of lipid peroxidation products (MDA and 4-HNE), HSC activation with attendant inflammation and fibrosis, and mutagenesis, and they interact with other aldehyde adducts to form stable hybrids that promote substantially greater degrees of injury, including DNA damage (Fig. 2).

Based on these observations, we hypothesize that acetaldehyde accumulation $\rightarrow$ oxidative stress, inflammation, cell injury $\rightarrow$ HSC activation and cytokine activation $\rightarrow$ ROS generation $\rightarrow$ radical ion accumulation $\rightarrow$ lipid peroxidation, DNA and protein adduct formation $\rightarrow$ protein and enzyme malfunction, impaired gene expression, and DNA damage $\rightarrow$ mutagenesis and fibrogenesis. In light of the potency of hybrid adducts with respect to accelerating and intensifying injury, it could be that "second hits" that perturbate metabolism and promote additional aldehyde accumulations and adducts may play pivotal roles in governing the path toward progressive hepatic fibrosis and cirrhosis versus hepatocellular carcinoma. In this regard, other disease states such as non-alcoholic fatty liver disease (NAFLD) or chronic hepatitis C virus infection, and ischemia/ hypoperfusion caused by heart failure or atherosclerosis result in increased accumulation of lipid peroxidation products and adduct formation. ${ }^{113,114}$ When combined with chronic alcohol abuse, these co-factors increase risk for progression to cirrhosis and/or HCC. ${ }^{115}$ Conditions that lead to increased iron deposition and consequently potentiate radical ion formation such as hemachromatosis, ${ }^{35}$ or ADH and ALDH gene polymorphisms that impair efficient metabolism of alcohol to carbon dioxide and water, could serve as co-factors in promoting end-stage liver disease or HCC. Finally, tobacco smoke contains nitrosamines that promote adduct formation, ${ }^{116}$ and since many alcohol abusers smoke or are exposed to smoke, they are at increased risk for generating adducts unrelated to acetaldehyde-associated adducts. Interactions between acetaldehyde and other adducts resulting in the formation of hybrid adducts may represent the pivotal factor governing long term consequences of chronic alcohol abuse with respect to the development of cirrhosis or HCC. Future investigations will need to focus on developing means to detect, characterize, and quantify hybrid adducts in liver, and identify approaches to limit their formation and accumulation in the context of chronic alcohol abuse.

\section{Acknowledgements}

Grants AA-11431, AA-12908, K24-AA-16126 from the National Institutes of Health and the Gastroenterology Foundation of South Africa supported this work. The authors also thank Mr. Rolf I. Carlson for generating the graphic artwork.

\section{References}

1. Voigt MD. Alcohol in hepatocellular cancer. Clin Liver Dis 2005; 9:151-69.

2. Druesne-Pecollo N, Tehard B, Mallet Y, Gerber M, Norat T, Hercberg S, et al. Alcohol and genetic polymorphisms: effect on risk of alcohol-related cancer. Lancet Oncol 2009; 10:173-80.

3. Seitz HK, Becker P. Alcohol metabolism and cancer risk. Alcohol Res Health 2007; 30:38-41, 4-7.

4. Mayans MV, Calvet X, Bruix J, Bruguera M, Costa J, Esteve J, et al. Risk factors for hepatocellular carcinoma in Catalonia, Spain. Int J Cancer 1990; 46:378-81.

5. Chuang SC, La Vecchia C, Boffetta P. Liver cancer: descriptive epidemiology and risk factors other than HBV and HCV infection. Cancer Lett 2009; 286:9-14.

6. Corrao G, Bagnardi V, Zambon A, La Vecchia C. A meta-analysis of alcohol consumption and the risk of 15 diseases. Prev Med 2004; 38:613-9.

7. Mills SJ, Harrison SA. Comparison of the natural history of alcoholic and nonalcoholic fatty liver disease. Curr Gastroenterol Rep 2005; 7:32-6.
8. Lau WY, Lai EC. Hepatocellular carcinoma: current management and recent advances. Hepatobiliary Pancreat Dis Int 2008; 7:237-57.

9. Verslype C, Van Cutsem E, Dicato M, Arber N, Berlin JD, Cunningham D, et al. The management of hepatocellular carcinoma. Current expert opinion and recommendations derived from the 10th World Congress on Gastrointestinal Cancer, Barcelona, 2008. Ann Oncol 2009; 20 Suppl 7:vii1-vii6.

10. Thiele GM, Klassen LW, Tuma DJ. Formation and immunological properties of aldehyde-derived protein adducts following alcohol consumption. Methods Mol Biol 2008; 447:235-57.

11. Tuma DJ, Casey CA. Dangerous byproducts of alcohol breakdown--focus on adducts. Alcohol Res Health 2003; 27:285-90.

12. Freeman TL, Tuma DJ, Thiele GM, Klassen LW, Worrall S, Niemela O, et al. Recent advances in alcohol-induced adduct formation. Alcohol Clin Exp Res 2005; 29:1310-6.
13. Barry RE, Williams AJ. Metabolism of ethanol and its consequences for the liver and gastrointestinal tract. Dig Dis 1988; 6:194-202.

14. Crabb DW, Liangpunsakul S. Acetaldehyde generating enzyme systems: roles of alcohol dehydrogenase, CYP2E1 and catalase, and speculations on the role of other enzymes and processes. Novartis Found Symp 2007; 285:4-16.

15. Kunitoh S, Imaoka S, Hiroi T, Yabusaki Y, Monna T, Funae Y. Acetaldehyde as well as ethanol is metabolized by human CYP2E1. J Pharmacol Exp Ther 1997; 280:527-32.

16. Brooks PJ, Theruvathu JA. DNA adducts from acetaldehyde: implications for alcohol-related carcinogenesis. Alcohol 2005; 35:187-93.

17. Niemela O. Acetaldehyde adducts in circulation. Novartis Found Symp 2007; 285:183-92; discussion 93-7.

18. Tuma DJ. Role of malondialdehyde-acetaldehyde adducts in liver injury. Free Radic Biol Med 2002; 32:303-8. 
19. Lieber CS. Alcoholic fatty liver: its pathogenesis and mechanism of progression to inflammation and fibrosis. Alcohol 2004; 34:9-19.

20. Homann N, Stickel F, Konig IR, Jacobs A, Junghanns $\mathrm{K}$, Benesova $\mathrm{M}$, et al. Alcohol dehydrogenase $1 \mathrm{C}^{*} 1$ allele is a genetic marker for alcohol-associated cancer in heavy drinkers. Int J Cancer 2006; 118:1998-2002.

21. Seitz HK, Stickel F. Risk factors and mechanisms of hepatocarcinogenesis with special emphasis on alcohol and oxidative stress. Biol Chem 2006; 387:349-60.

22. Seitz HK, Stickel F. Molecular mechanisms of alcoholmediated carcinogenesis. Nat Rev Cancer 2007; 7:599612.

23. Munaka M, Kohshi K, Kawamoto T, Takasawa S, Nagata N, Itoh $\mathrm{H}$, et al. Genetic polymorphisms of tobacco- and alcohol-related metabolizing enzymes and the risk of hepatocellular carcinoma. J Cancer Res Clin Oncol 2003; 129:355-60.

24. Ohhira M, Fujimoto Y, Matsumoto A, Ohtake T, Ono M, Kohgo Y. Hepatocellular carcinoma associated with alcoholic liver disease: a clinicopathological study and genetic polymorphism of aldehyde dehydrogenase 2 . Alcohol Clin Exp Res 1996; 20:378-82.

25. Barry RE. Role of acetaldehyde in the pathogenesis of alcoholic liver disease. Br J Addict 1988; 83:1381-6.

26. Marietta C, Thompson LH, Lamerdin JE, Brooks PJ. Acetaldehyde stimulates FANCD2 monoubiquitination, H2AX phosphorylation, and BRCA1 phosphorylation in human cells in vitro: implications for alcoholrelated carcinogenesis. Mutat Res 2009; 664:77-83.

27. Poschl G, Seitz HK. Alcohol and cancer. Alcohol Alcohol 2004; 39:155-65.

28. Seitz HK, Cho CH. Contribution of alcohol and tobacco use in gastrointestinal cancer development. Methods Mol Biol 2009; 472:217-41.

29. Cederbaum AI, Lu Y, Wu D. Role of oxidative stress in alcohol-induced liver injury. Arch Toxicol 2009; 83:519-48.

30. Wu D, Cederbaum AI. Oxidative stress and alcoholic liver disease. Semin Liver Dis 2009; 29:141-54.

31. Nagata K, Suzuki H, Sakaguchi S. Common pathogenic mechanism in development progression of liver injury caused by non-alcoholic or alcoholic steatohepatitis. J Toxicol Sci 2007; 32:453-68.

32. Purohit V, Bode JC, Bode C, Brenner DA, Choudhry MA, Hamilton F, et al. Alcohol, intestinal bacterial growth, intestinal permeability to endotoxin, and medical consequences: summary of a symposium. Alcohol 2008; 42:349-61.

33. Rao RK. Acetaldehyde-induced barrier disruption and paracellular permeability in Caco-2 cell monolayer. Methods Mol Biol 2008; 447:171-83.

34. Bonkovsky HL, Lambrecht RW. Iron-induced liver injury. Clin Liver Dis 2000; 4:409-29, vi-vii.

35. Kew MC. Hepatic iron overload and hepatocellular carcinoma. Cancer Lett 2009; 286:38-43.

36. Asare GA, Bronz M, Naidoo V, Kew MC. Synergistic interaction between excess hepatic iron and alcohol ingestion in hepatic mutagenesis. Toxicology 2008; 254:11-8.

37. Brooks PJ. DNA damage, DNA repair, and alcohol toxicity--a review. Alcohol Clin Exp Res 1997; 21:1073-82.

38. Biewald J, Nilius R, Langner J. Occurrence of acetaldehyde protein adducts formed in various organs of chronically ethanol fed rats: an immunohistochemical study. Int J Mol Med 1998; 2:389-96.

39. Seitz HK, Meier P. The role of acetaldehyde in upper digestive tract cancer in alcoholics. Transl Res 2007; 149:293-7.

40. Collier JD, Bassendine MF, Burt AD, Major GN. Characterisation of the DNA repair enzyme for $\mathrm{O}(6)$ methylguanine in cirrhosis. J Hepatol 1996; 25:158-65.

41. Tuma DJ, Smith SL, Sorrell MF. Acetaldehyde and microtubules. Ann NY Acad Sci 1991; 625:786-92.

42. Jukkola A, Niemela O. Covalent binding of acetaldehyde to type III collagen. Biochem Biophys Res Commun 1989; 159:163-9.
43. Niemela O. Aldehyde-protein adducts in the liver as a result of ethanol-induced oxidative stress. Front Biosci 1999; 4:506-13

44. Sabol DA, Basista MH, Brecher AS, Haider K, Kleshinski J. Coagulation protein function VII: diametric effects of acetaldehyde on factor VII and factor IX function. Dig Dis Sci 1999; 44:2564-7.

45. Mauch TJ, Donohue TM, Jr., Zetterman RK, Sorrell MF, Tuma DJ. Covalent binding of acetaldehyde selectively inhibits the catalytic activity of lysine-dependent enzymes. Hepatology 1986; 6:263-9.

46. Michael Lieberman AM. Marks' basic medical biochemistry: a clinical approach. Philadelphia: Lippincott Williams \& Wilkins, 2008.

47. Albano E. Free radical mechanisms in immune reactions associated with alcoholic liver disease. Free Radic Biol Med 2002; 32:110-4.

48. Fang JL, Vaca CE. Detection of DNA adducts of acetaldehyde in peripheral white blood cells of alcohol abusers. Carcinogenesis 1997; 18:627-32.

49. Matsuda T, Yabushita H, Kanaly RA, Shibutani S, Yokoyama A. Increased DNA damage in ALDH2 deficient alcoholics. Chem Res Toxicol 2006; 19:1374-8.

50. Nagayoshi H, Matsumoto A, Nishi R, Kawamoto T, Ichiba M, Matsuda T. Increased formation of gastric $\mathrm{N}$ (2)-ethylidene-2'-deoxyguanosine DNA adducts in aldehyde dehydrogenase- 2 knockout mice treated with ethanol. Mutat Res 2009; 673:74-7.

51. Balbo S, Hashibe M, Gundy S, Brennan P, Canova C, Simonato L, et al. N2-ethyldeoxyguanosine as a potential biomarker for assessing effects of alcohol consumption on DNA. Cancer Epidemiol Biomarkers Prev 2008; 17:3026-32.

52. Lu Y, Cederbaum AI. CYP2E1 and oxidative liver injury by alcohol. Free Radic Biol Med 2008; 44:723-38.

53. Cederbaum AI. CYP2E1--biochemical and toxicological aspects and role in alcohol-induced liver injury. $\mathrm{Mt}$ Sinai J Med 2006; 73:657-72.

54. Breen AP, Murphy JA. Reactions of oxyl radicals with DNA. Free Radic Biol Med 1995; 18:1033-77.

55. Warnakulasuriya $S$, Parkkila $S$, Nagao T, Preedy VR, Pasanen M, Koivisto $\mathrm{H}$, et al. Demonstration of ethanol-induced protein adducts in oral leukoplakia (pre-cancer) and cancer. J Oral Pathol Med 2008; 37:157-65.

56. Niemela O. Distribution of ethanol-induced protein adducts in vivo: relationship to tissue injury. Free Radic Biol Med 2001; 31:1533-8.

57. Hussain SP, Raja K, Amstad PA, Sawyer M, Trude LJ, Wogan GN, et al. Increased p 53 mutation load in nontumorous human liver of wilson disease and hemochromatosis: oxyradical overload diseases. Proc Natl Acad Sci USA 2000; 97:12770-5.

58. Tuma DJ, Thiele GM, Xu D, Klassen LW, Sorrell MF Acetaldehyde and malondialdehyde react together to generate distinct protein adducts in the liver during long-term ethanol administration. Hepatology 1996; 23:872-80.

59. Li CJ, Nanji AA, Siakotos AN, Lin RC. Acetaldehydemodified and 4-hydroxynonenal-modified proteins in the livers of rats with alcoholic liver disease. Hepatology 1997; 26:650-7.

60. Niemela O, Parkkila S, Pasanen M, Iimuro Y, Bradford B, Thurman RG. Early alcoholic liver injury: formation of protein adducts with acetaldehyde and lipid peroxidation products, and expression of CYP2E1 and CYP3A. Alcohol Clin Exp Res 1998; 22:2118-24.

61. Holstege A, Bedossa P, Poynard T, Kollinger M, Chaput JC, Houglum K, et al. Acetaldehyde-modified epitopes in liver biopsy specimens of alcoholic and nonalcoholic patients: localization and association with progression of liver fibrosis. Hepatology 1994; 19:367-74.

62. MacSween RNM. Pathology of the liver. Churchill Livingstone, 2002.
63. Niemela O, Parkkila S, Yla-Herttuala S, Villanueva J, Ruebner B, Halsted CH. Sequential acetaldehyde production, lipid peroxidation, and fibrogenesis in micropig model of alcohol-induced liver disease. Hepatology 1995; 22:1208-14.

64. Niemela O, Parkkila S, Yla-Herttuala S, Halsted C, Witztum JL, Lanca A, et al. Covalent protein adducts in the liver as a result of ethanol metabolism and lipid peroxidation. Lab Invest 1994; 70:537-46.

65. Paradis V, Scoazec JY, Kollinger M, Holstege A, Moreau A, Feldmann G, et al. Cellular and subcellular localization of acetaldehyde-protein adducts in liver biopsies from alcoholic patients. J Histochem Cytochem 1996. 44:1051-7.

66. Duryee MJ, Klassen LW, Jones BL, Willis MS, Tuma DJ, Thiele GM. Increased immunogenicity to P815 cells modified with malondialdehyde and acetaldehyde. Int Immunopharmacol 2008; 8:1112-8.

67. Latvala J, Hietala J, Koivisto H, Jarvi K, Anttila P, Niemela O. Immune Responses to Ethanol Metabolites and Cytokine Profiles Differentiate Alcoholics with or without Liver Disease. Am J Gastroenterol 2005; 100:1303-10.

68. Viitala K, Israel Y, Blake JE, Niemela O. Serum IgA, IgG, and $\mathrm{IgM}$ antibodies directed against acetaldehyde-derived epitopes: relationship to liver disease severity and alcohol consumption. Hepatology 1997; 25:1418-24.

69. Worrall S, de Jersey J, Wilce PA, Seppa K, Hurme L, Sillanaukee P. Relationship between alcohol intake and immunoglobulin a immunoreactivity with acetaldehyde-modified bovine serum albumin. Alcohol Clin Exp Res 1996; 20:836-40.

70. Yokoyama H, Ishii H, Nagata S, Kato S, Kamegaya K, Tsuchiya M. Experimental hepatitis induced by ethanol after immunization with acetaldehyde adducts. Hepatology 1993; 17:14-9.

71. Yokoyama $H$, Nagata $S$, Moriya $S$, Kato $S$, Ito $T$, Kamegaya K, et al. Hepatic fibrosis produced in guinea pigs by chronic ethanol administration and immunization with acetaldehyde adducts. Hepatology 1995; 21:1438-42.

72. Hietala J, Koivisto H, Latvala J, Anttila P, Niemela O. IgAs against acetaldehyde-modified red cell protein as a marker of ethanol consumption in male alcoholic subjects, moderate drinkers, and abstainers. Alcohol Clin Exp Res 2006; 30:1693-8.

73. Brenner DA. Molecular pathogenesis of liver fibrosis. Trans Am Clin Climatol Assoc 2009; 120:361-8.

74. Kharbanda KK, Todero SL, Shubert KA, Sorrell MF, Tuma DJ. Malondialdehyde-acetaldehyde-protein adducts increase secretion of chemokines by rat hepatic stellate cells. Alcohol 2001; 25:123-8.

75. Jarnagin WR, Rockey DC, Koteliansky VE, Wang SS, Bissell DM. Expression of variant fibronectins in wound healing: cellular source and biological activity of the EIIIA segment in rat hepatic fibrogenesis. J Cell Biol 1994; 127:2037-48.

76. Thiele GM, Worrall S, Tuma DJ, Klassen LW, Wyatt TA, Nagata N. The chemistry and biological effects of malondialdehyde-acetaldehyde adducts. Alcohol Clin Exp Res 2001; 25:218S-24S.

77. Mello T, Ceni E, Surrenti C, Galli A. Alcohol induced hepatic fibrosis: role of acetaldehyde. Mol Aspects Med 2008; 29:17-21.

78. Ma X, Svegliati-Baroni G, Poniachik J, Baraona E, Lieber CS. Collagen synthesis by liver stellate cells is released from its normal feedback regulation by acetaldehyde-induced modification of the carboxyl-terminal propeptide of procollagen. Alcohol Clin Exp Res 1997; 21:1204-11.

79. Palomo I, Alarcon M, Moore-Carrasco R, Argiles JM. Hemostasis alterations in metabolic syndrome (review) Int J Mol Med 2006; 18:969-74.

80. Hill GE, Miller JA, Baxter BT, Klassen LW, Duryee MJ, Tuma DJ, et al. Association of malondialdehyde-acetaldehyde (MAA) adducted proteins with atheroscleroticinduced vascular inflammatory injury. Atherosclerosis 1998; 141:107-16. 
81. Redmond EM, Morrow D, Kundimi S, MillerGraziano CL, Cullen JP. Acetaldehyde stimulates monocyte adhesion in a P-selectin- and TNFalphadependent manner. Atherosclerosis 2009; 204:372-80.

82. Strom A, Fredrikson GN, Schiopu A, Ljungcrantz I, Soderberg I, Jansson B, et al. Inhibition of injury-induced arterial remodelling and carotid atherosclerosis by recombinant human antibodies against aldehyde-modified apoB-100. Atherosclerosis 2007; 190:298-305.

83. van Leeuwen M, Damoiseaux J, Duijvestijn A, Tervaert JW. The therapeutic potential of targeting $B$ cells and anti-oxLDL antibodies in atherosclerosis. Autoimmun Rev 2009; 9:53-7.

84. Vindis C, Escargueil-Blanc I, Elbaz M, Marcheix B, Grazide MH, Uchida K, et al. Desensitization of platelet-derived growth factor receptor-beta by oxidized lipids in vascular cells and atherosclerotic lesions: prevention by aldehyde scavengers. Circ Res 2006; 98:785-92.

85. Cai L. Alcoholic cardiomyopathy: acetaldehyde, insulin insensitization and ER stress. J Mol Cell Cardiol 2008; 44:979-82.

86. Laonigro I, Correale M, Di Biase M, Altomare E. Alcohol abuse and heart failure. Eur J Heart Fail 2009; 11:453-62.

87. Ren J. Acetaldehyde and alcoholic cardiomyopathy: lessons from the $\mathrm{ADH}$ and ALDH2 transgenic models. Novartis Found Symp 2007; 285:69-76.

88. Ren J, Wold LE. Mechanisms of alcoholic heart disease. Ther Adv Cardiovasc Dis 2008; 2:497-506.

89. Zhang X, Li SY, Brown RA, Ren J. Ethanol and acetaldehyde in alcoholic cardiomyopathy: from bad to ugly en route to oxidative stress. Alcohol 2004; 32:175-86.

90. Preedy VR, Siddiq T, Why HJ, Richardson PJ. Ethanol toxicity and cardiac protein synthesis in vivo. Am Heart J 1994; 127:1432-9.

91. Aberle NS, 2nd, Ren J. Short-term acetaldehyde exposure depresses ventricular myocyte contraction: role of cytochrome P450 oxidase, xanthine oxidase, and lipid peroxidation. Alcohol Clin Exp Res 2003; 27:577-83.

92. Schoppet M, Maisch B. Alcohol and the heart. Herz 2001; 26:345-52.

93. Budas GR, Disatnik MH, Mochly-Rosen D. Aldehyde dehydrogenase 2 in cardiac protection: a new therapeutic target? Trends Cardiovasc Med 2009; 19:158-64.
94. Ma H, Li J, Gao F, Ren J. Aldehyde dehydrogenase 2 ameliorates acute cardiac toxicity of ethanol: role of protein phosphatase and forkhead transcription factor. J Am Coll Cardiol 2009; 54:2187-96.

95. Xu F, Chen Y, Lv R, Zhang H, Tian H, Bian Y, et al. ALDH2 genetic polymorphism and the risk of type II diabetes mellitus in CAD patients. Hypertens Res; 33:49-55.

96. Doser TA, Turdi S, Thomas DP, Epstein PN, Li SY, Ren J. Transgenic overexpression of aldehyde dehydrogenase-2 rescues chronic alcohol intake-induced myocardial hypertrophy and contractile dysfunction. Circulation 2009; 119:1941-9.

97. Guo R, Zhong L, Ren J. Overexpression of aldehyde dehydrogenase-2 attenuates chronic alcohol exposureinduced apoptosis, change in Akt and Pim signalling in liver. Clin Exp Pharmacol Physiol 2009; 36:463-8.

98. Li SY, Ren J. Cardiac overexpression of alcohol dehydrogenase exacerbates chronic ethanol ingestion induced myocardial dysfunction and hypertrophy: role of insulin signaling and ER stress. J Mol Cell Cardiol 2008; 44:992-1001.

99. Brunt EM. Alcoholic and nonalcoholic steatohepatitis. Clin Liver Dis 2002; 6:399-420, vii.

100. Page JM, Harrison SA. NASH and HCC. Clin Liver Dis 2009; 13:631-47.

101. Baou K, Vlachopoulos C, Manesis E, Archimandritis A, Stefanadis C. Non-alcoholic fatty liver and cardiovascular disease: an emerging relationship. Hellenic J Cardiol 2007; 48:37-41.

102. Edens MA, Kuipers F, Stolk RP. Non-alcoholic fatty liver disease is associated with cardiovascular disease risk markers. Obes Rev 2009; 10:412-9.

103. Haffner SM. Relationship of metabolic risk factors and development of cardiovascular disease and diabetes. Obesity 2006; 14:121S-7S.

104. Targher G. Non-alcoholic fatty liver disease, the metabolic syndrome and the risk of cardiovascular disease: the plot thickens. Diabet Med 2007; 24:1-6.

105. Bloomer RJ, Fisher-Wellman KH. Systemic oxidative stress is increased to a greater degree in young, obese women following consumption of a high fat meal. Oxid Med Cell Longev 2009; 2:19-25.
106. Willis MS, Thiele GM, Tuma DJ, Klassen LW. T cell proliferative responses to malondialdehyde-acetaldehyde haptenated protein are scavenger receptor mediated. Int Immunopharmacol 2003; 3:1381-99.

107. Koivisto H, Hietala J, Anttila P, Parkkila S, Niemel O. Long-term ethanol consumption and macrocytosis: diagnostic and pathogenic implications. J Lab Clin Med 2006; 147:191-6.

108. Latvala J, Parkkila S, Melkko J, Niemela O. Acetaldehyde adducts in blood and bone marrow of patients with ethanol-induced erythrocyte abnormalities. Mol Med 2001; 7:401-5.

109. Baraona E, Di Padova C, Tabasco J, Lieber CS. Red blood cells: a new major modality for acetaldehyde transport from liver to other tissues. Life Sci 1987; 40:253-8.

110. Brecher AS, Hommema EL. The influence of acetal dehyde and glycosaminoglycans upon factor $\mathrm{Xa}$ - and factor X-deficient plasma. Can J Physiol Pharmacol 2002; 80:879-86

111. Brecher AS, Moon AR, Gray KD. The effect of acetaldehyde-glycosaminoglycan mixtures upon Factor IXa and Factor IX-Deficient Plasma. Alcohol 2006; 39:97-104.

112. Suchocki EA, Brecher AS. The effect of acetaldehyde on human plasma factor XIII function. Dig Dis Sci 2007; 52:3488-92.

113. Fierbinteanu-Braticevici C, Mohora M, Cretoiu D, Cretoiu S, Petrisor A, Usvat R, et al. Role of oxidative stress in the pathogenesis of chronic hepatitis $\mathrm{C}$ (CHC). Rom J Morphol Embryol 2009; 50:407-12.

114. Tessari P, Coracina A, Cosma A, Tiengo A. Hepatic lipid metabolism and non-alcoholic fatty liver disease. Nutr Metab Cardiovasc Dis 2009; 19:291-302.

115. Fattovich G, Stroffolini T, Zagni I, Donato F. Hepatocellular carcinoma in cirrhosis: incidence and risk factors. Gastroenterology 2004; 127:S35-50.

116. Hecht SS. Progress and challenges in selected areas of tobacco carcinogenesis. Chem Res Toxicol 2008; 21:160-71. 


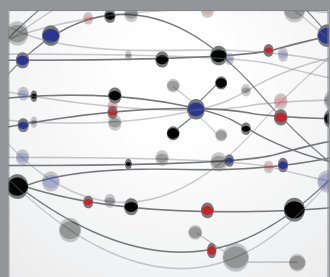

The Scientific World Journal
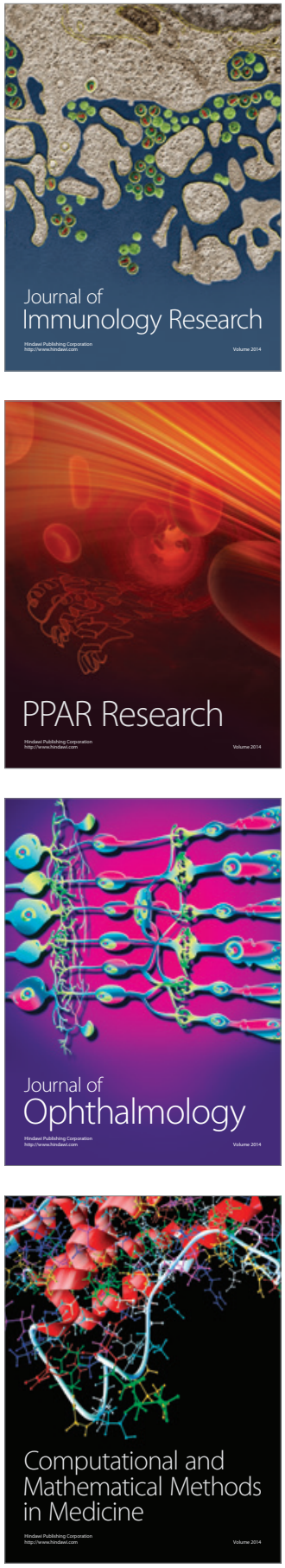

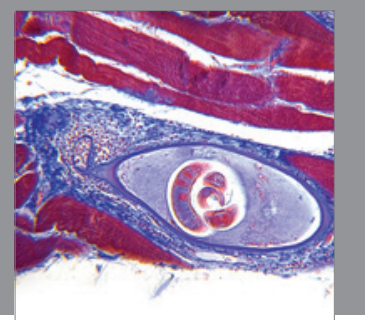

Gastroenterology

Research and Practice
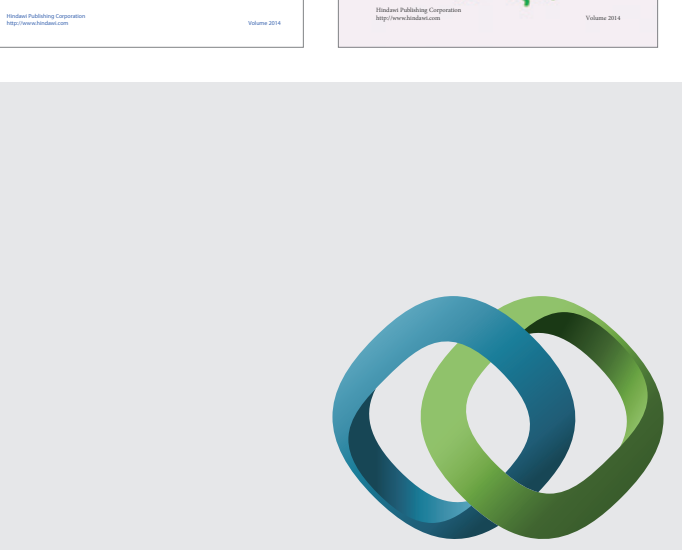

\section{Hindawi}

Submit your manuscripts at

http://www.hindawi.com
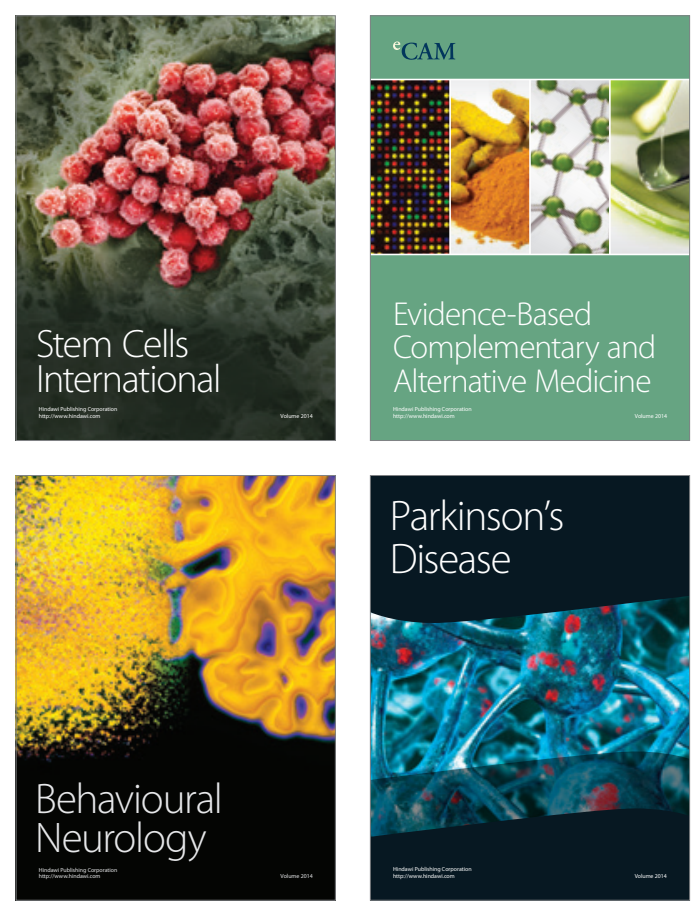

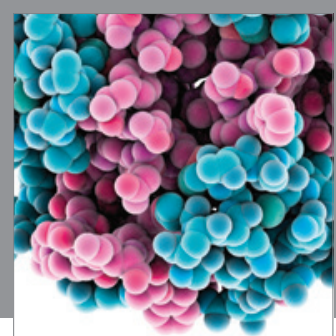

Journal of
Diabetes Research

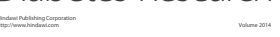

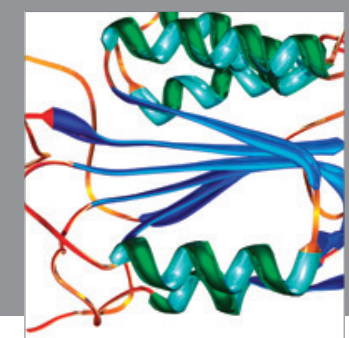

Disease Markers
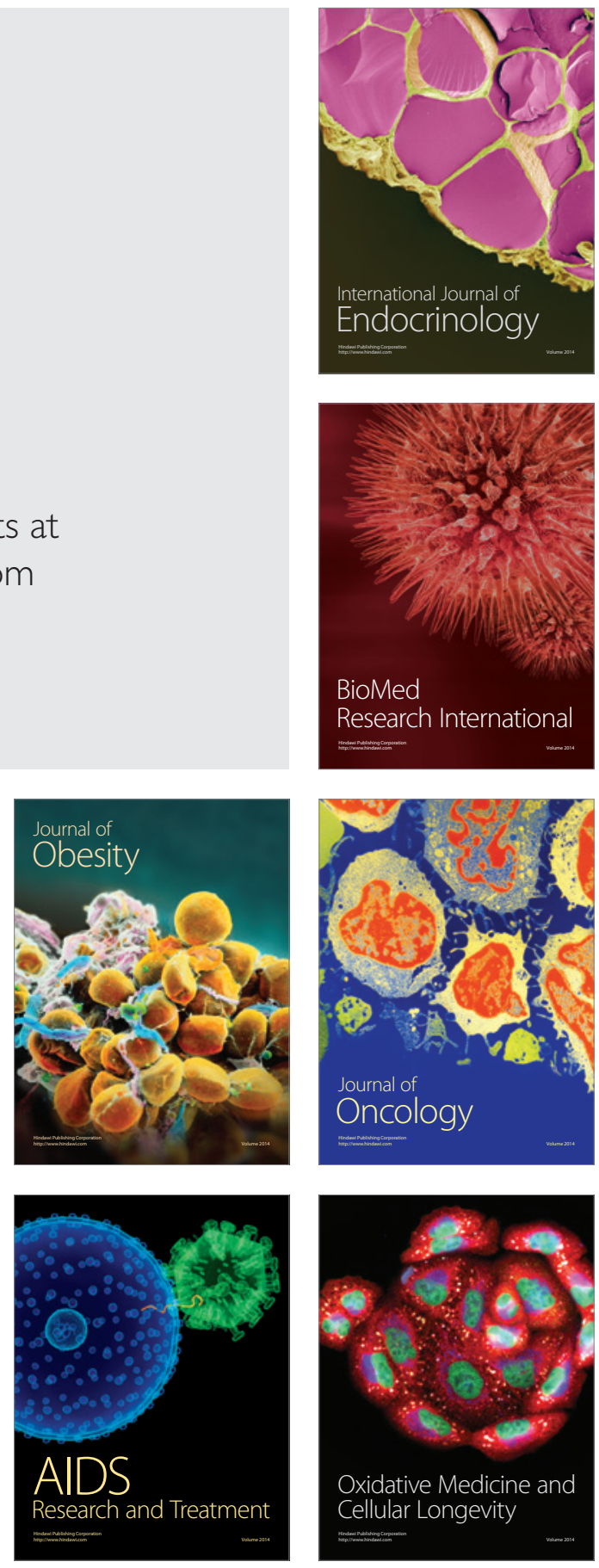